\title{
Adaptive Sliding Mode Control of Autonomous Underwater Vehicles in the Dive Plane
}

\author{
ROBERTO CRISTI, MEMBER, IEEE, FOTIS A. PAPOULIAS, AND ANTHONY J. HEALEY
}

\begin{abstract}
The problem of controlling an Autonomous Underwater Vehicle (AUV) in a diving maneuver is addressed. The requirement for having a simple controller which performs satisfactorily in the presence of dynamical uncertainties calls for a design using the sliding mode approach, based on a dominant linear model and bounds on the nonlinear perturbations of the dynamics. Both nonadaptive and adaptive techniques are considered, leading to the design of robust controllers that can adjust to the changing dynamics and operating conditions. Also, the problem of using the observed state in the control design is addressed, leading to a sliding mode control system based on input-output signals in terms of dive-plane command and depth measurement. Numerical simulations using a full set of nonlinear equations of motion show the effectiveness of the proposed techniques.
\end{abstract}

\section{INTRODUCTION}

M ANEUVERING in the dive plane, depth changing and depth keeping are essential performance requirements for any Autonomous Underwater Vehicle (AUV). Vehicle response depends heavily on its particular design and configuration, operating conditions, and environmental forces. While questions pertaining to fluid-body interaction and reliable prediction of hydrodynamic forces are still the subject of current research, design and installation of AUV controllers is a matter of immediate need. This is in response to AUV's being recognized as an alternative to manned submarines for a variety of underwater missions at minimal capital and no manpower expense.

Any automatic controller design for an AUV must satisfy two conflicting requirements: First, it has to be sophisticated enough to perform its mission in the realm of complicated and ever-changing vehicle/environment interactions; secondly, it has to be simple enough so that on-line implementation is possible by the onboard vehicle computer at a sufficiently high sample rate.

This paper attempts an attack at the problem of the AUV dive-plane response. Section I presents two dynamical models for the AUV response in the vertical plane. A simple, linear model is used for control law design, whereas a more complicated nonlinear model is reserved for visual simulation and demonstration. The difference between the models simulates the differences that exist between any vehicle model and the real world. Section II is devoted to the control design using

Manuscript revised March 27, 1990. This work was supported by the NPS Direct Research Fund.

R. Cristi is with the Department of Electrical and Computer Engineering, Naval Postgraduate School, Monterey, CA 93943.

F. A. Papoulias and A. J. Healey are with the Department of Mechanical Engineering, Naval Postgraduate School, Monterey, CA 93943.

IEEE Log Number 9036353. sliding modes, while Section III presents an adaptive version which compensates for uncertainties of the linearized dynamics. The issue of designing a sliding mode controller using estimated states is presented in Section IV. Numerical simulation results from a three-dimensional real-time simulation of the AUV are given in Section V, where vehicle motion is dynamically simulated from the complete set of equations of motion.

\section{Equations of Motions}

An exact set of equations of motion for a rigid body moving in an ideal fluid can, at least in principle, be derived from the Kelvin-Kirchhoff hydromechanical equations. Such a formidable formulation, invaluable as it may be for force prediction and motion analysis, presents some technical drawbacks from the point of view of on-line control system design. Namely, the required computational time is so extensive that with today's technology it is not possible that the necessary calculations could be performed by the onboard vehicle computer at a sufficiently high sample rate. For this reason, various simplified models have been developed and used in the study and control of motions of underwater bodies. Assuming that the vehicle motions are relatively slow, an assumption which is valid for almost all usual maneuvers, the dynamics of the AUV can be described by a set of 12 nonlinear, coupled, first-order differential equations with constant coefficients [1]. Several studies at the Naval Postgraduate School (NPS) [2] were based on a similar nonlinear model of the Swimmer Delivery Vehicle (SDV) designed at NCSC (Naval Coastal Systems Center) for which a complete set of hydrodynamic derivatives exists [3].

Restricting our attention to the dive plane, the equations of motion of a symmetrical body with horizontal plane-control surfaces at zero become:

$$
\begin{aligned}
m(\dot{u}+q w)= & \frac{\rho}{2} l^{4} X_{q q} q^{2}+\frac{\rho}{2} l^{3}\left(X_{\dot{u}} \dot{u}+X_{w q} w q+X_{q \delta} u q \delta\right. \\
& +\frac{\rho}{2} l^{2}\left(X_{w w} w^{2}+X_{w \delta} \delta u w\right)+\frac{\rho}{2} l^{2} u^{2} X_{\delta \delta} \delta^{2} \\
& -(W-B) \sin \theta+F_{\text {PROPULSION }} \\
m(\dot{w}-u q)= & \frac{\rho}{2} l^{4} Z_{\dot{q}} \dot{q}+\frac{\rho}{2} l^{3}\left(Z_{\dot{w}} \dot{w}+Z_{q} u q\right) \\
& +\frac{\rho}{2} l^{2}\left(Z_{w} u w+u^{2} Z_{\delta} \delta\right) \\
& +(W-B) \cos \theta+Z_{\mathrm{HEAVE}}
\end{aligned}
$$

U.S. Government work not protected by U.S. copyright 


$$
\begin{aligned}
I_{y} \dot{q}= & \frac{\rho}{2} l^{5} M_{\dot{q}} \dot{q}+\frac{\rho}{2} l^{4}\left(M_{\dot{w}} \dot{w}+M_{q} u q\right) \\
& +\frac{\rho}{2} l^{3}\left(M_{w} u w+u^{2} M_{\delta} \delta\right. \\
& -\left(z_{G} W-z_{B} B\right) \sin \theta+M_{\mathrm{PITCH}} \\
\dot{\theta}= & q \\
\dot{z}= & -u \sin \theta+w \cos \theta
\end{aligned}
$$

where the standard notation of [1] is adopted, and $Z_{\text {HEAVE }}$ and $M_{\text {PITCH }}$ represent the cross-flow drag terms [3]. Although these equations can be significantly simplified [4], they appear still to be very complex for this study.

Control of plants with such nonlinear and coupled models has been traditionally accomplished by linear controllers that may or may not be gain-scheduled. Recent work with sliding mode control [5] has been extremely promising because of added robustness inherent in the method. The most wellestablished approach is based on a canonical form of the plant dynamics and nonlinear state feedback. However, a difficulty arises in the direct application of the approach in [5] to (1), since these equations are coupled, nonlinear, and not in the canonical form:

$$
x^{n}(t)=f\left(x^{n-1}, \cdots, x^{1}, x, t, u\right)
$$

on which most of the sliding mode control approaches [5], [6] are based.

A way to circumvent this problem while maintaining the robustness features of the sliding mode controller is to base the design on an approximate model, linearized around nominal flight-operating conditions. Assuming that the vehicle maintains forward motion (as opposed to the hovering mode of ROV operations), nominal conditions are determined on the basis of the nominal speed $\bar{U}_{0}$, pitch rate $q$, pitch $\theta$, and depth $z$ signals. For the submersible under consideration, the heave velocity $w$ has a negligible effect on $q$ and $\theta$ and can be considered as a disturbance acting on the depth $z$. Extensive water testing [9] confirmed the validity of this assumption.

As a consequence, we can separate the dynamic model (1) into its linear component and nonlinear state-dependent disturbance as

$$
\dot{x}=\left[\begin{array}{ccc}
-a_{1} & -a_{2} & 0 \\
1 & 0 & 0 \\
0 & -\bar{U}_{0} & 0
\end{array}\right] \boldsymbol{x}+\left[\begin{array}{l}
b \\
0 \\
0
\end{array}\right] \delta+f(x)
$$

where the term $f(x)$ denotes the deviation from linearity. The linear part of the model is shown in Fig. 1(a), where the feedback term $K$ accounts for the restoring hydrostatic moment of the centers of gravity and buoyancy being displaced by a change in the pitch angle.

The parameters of a linear model can now be estimated by a standard recursive least squares or an instrumental variable algorithm by assuming an ARX discrete time model:

$$
A\left(q^{-1}\right) y(k T)=B\left(q^{-1}\right) u(k T)+e(k T)
$$
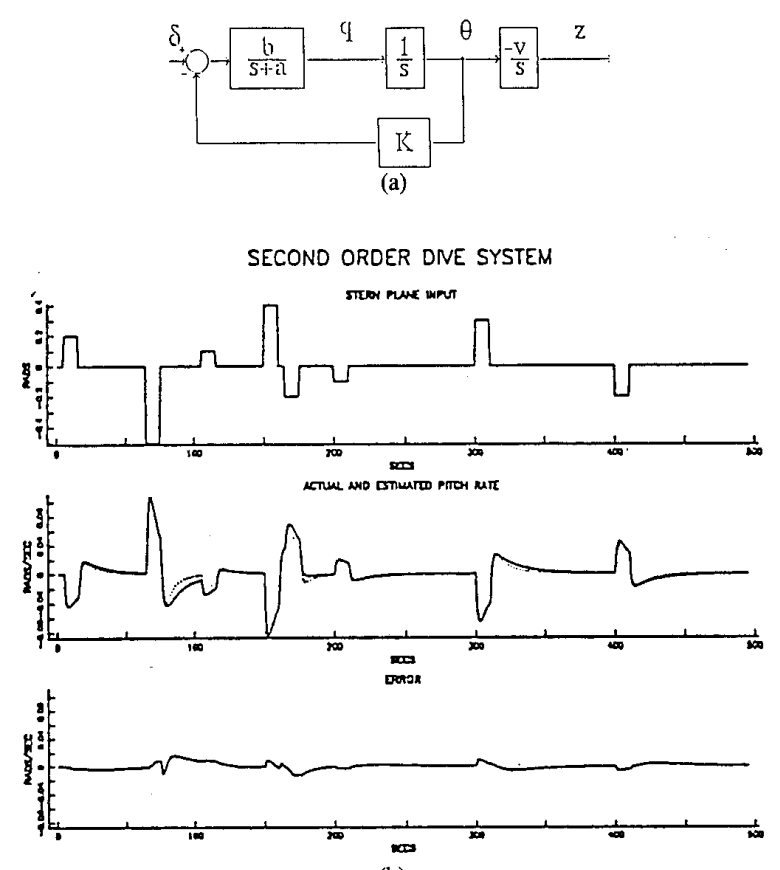

(b)

Fig. 1. (a) Linear model. (b) Pitch rate (actual and modeled).

$A, B$ being polynomials in the time-delay operator $\left(q^{-1} y(k T)=y(k T-T), T\right.$ being the sampling interval), and $e$ a random error sequence, possibly colored. The output of the linear (estimated) and nonlinear models' pitch rate is shown in Fig. 1(b) for a twin screw vehicle of $17.4 \mathrm{ft}$ in length, weighing $1200 \mathrm{lbs}$, and operating at $500 \mathrm{rpm}$. Also, the estimated dynamics at different operating conditions in terms of estimated poles and zeros in the $z$ plane are shown in Table I. The effect of the restoring moment (gain $K$ ) being more dominant at lower speeds can be seen from the poles becoming complex.

\section{Sliding Mode Control Design}

It is evident from the discussion in Section II that any vehicle description based on a set of differential equations of motion can only be approximate in nature.

The necessity of coping with uncertainties in the model calls for a robust control input $u$ of the form:

$$
u=\hat{u}+\bar{u}
$$

where $\hat{u}$ is determined on the basis of the nominal model, while $\bar{u}$ compensates for deviations from ideal performances due to uncertainties.

In current approaches to the control of a wide class of nonlinear plants, the nominal control term $\hat{u}$ is determined by the full nonlinear model, in our case equation (1). This is the case found in computed torque techniques applied to the control of mechanical manipulators [7]. A similar approach of interest to our work has been presented for the control of submersibles [5], [6].

In our research, by contrast, the nominal model is based 
TABLE I

Estimated Discrete-Time Dynamics of the AUV

\begin{tabular}{c|cc|c}
\hline Dire Model & Parameters & $\mathrm{H}(\mathrm{z})$ \\
\hline $500 \mathrm{rpm}$ & $a_{1}=1.613$ & $b_{1}=-0.0024$ & $\frac{(z+16.5)}{(z-0.967)(z-0.645)}$ \\
& $a_{2}=-0.624$ & $b_{2}=0.0402$ & \\
\hline $300 \mathrm{rpm}$ & $a_{1}=1.745$ & $b_{1}=-0.001$ & $\frac{(z+15.9)}{(z-0.9-15)(z-0.8)}$ \\
& $a_{2}=-0.756$ & $b_{2}=-0.016$ & \\
\hline \multirow{3}{*}{$100 \mathrm{rpm}$} & $a_{1}=1.922$ & $b_{1}=-0.0006$ & $\frac{(z+3.56)}{(z-0.961 \pm 0.072 j)}$ \\
& $a_{2}=-0.928$ & $b_{2}=-0.0023$ & \\
\hline
\end{tabular}

on the linearized dynamics around the operating conditions of the vehicle and changes with nominal speed. Several reasons are at the basis of this choice: (i) Computer simulations [8] and experimental verification in water testing [9] show that at constant speeds the linear model is indeed a good approximation of the nonlinear dynamics; (ii) for a linear model, the nominal control input $\hat{u}$ can be determined using robust linear control techniques such as pole placement and LQG; (iii) when the state signals are not available, they can be estimated by an observer based on the linear dynamics, as discussed in a later section and in a forthcoming paper [10].

The term $\bar{u}$ coping with model uncertainties can be determined on the basis of a sliding mode control [11], [12] approach. It is well known that sliding mode control provides effective and robust ways of controlling uncertain plants [11], [13] by means of a switching control law which drives the plant's state trajectory onto a user-chosen surface (the sliding surface) in the state space. The plant's state trajectory is maintained on this surface for all subsequent times.

The only restriction on the choice of the sliding surface

$$
\sigma(x)=0
$$

is that it has to be associated with stable dynamics in the sense that

$$
\sigma(x(t))=0, \quad \text { for all } t>t_{0} \Rightarrow \quad \lim _{t \rightarrow \infty} x(t)=0
$$

for any initial conditions $\boldsymbol{x}\left(t_{0}\right)$. The choice of a linear sliding surface:

$$
\sigma(x)=s^{T} x
$$

for some vector $s \in R^{3}$ allows the use of pole-placement techniques in the design of the nonlinear controller.

By defining the Lyapunov function:

$$
V(x)=\frac{1}{2}[\sigma(x)]^{2}
$$

we guarantee that the sliding surface $\sigma(x)=0$ is reached in a finite amount of time by the condition:

$$
\sigma \dot{\sigma}=-\eta_{0}^{2}(x)|\sigma(x)| \quad \text { or } \quad \dot{\sigma}=-\eta_{0}^{2}(x) \operatorname{sign}(\sigma) \text {. }
$$

Since $\sigma(x)=s^{T} x$, we can use (8) and (2) to get:

$$
s^{T}(A x+b u+f)=-\eta_{0}^{2}(x) \operatorname{sign}(\sigma) .
$$

By knowing a bound $\eta$ on the nonlinearity such that

$$
\eta^{2}(x)>\frac{\left|s^{T} f(x)\right|}{\left|s^{T} b\right|}
$$

for all $x$, the condition (8) with $\eta_{0}^{2}=\eta^{2}-s^{T} f$ is satisfied by chosing the control input:

$$
\begin{aligned}
& u=-\left(s^{T} b\right)^{-1} s^{T} A x-\eta^{2}\left(s^{T} b\right)^{-1} \operatorname{sign}(\sigma) \quad \text { or } \\
& u=\hat{u}+\bar{u} .
\end{aligned}
$$

As mentioned at the beginning of this section, it is important to recognize that the feedback control law $u$ is composed of two parts. The first,

$$
\hat{u}=-\left(s^{T} b\right)^{-1} s^{T} A \boldsymbol{x}
$$

is a linear feedback law based on the nominal model (1.2), whereas the second,

$$
\bar{u}=-\eta^{2}\left(\boldsymbol{s}^{T} b\right)^{-1} \operatorname{sign}(\sigma)
$$

is a nonlinear feedback with its sign toggling between plus and minus according to which side of the sliding plane the system is located in. Two comments are in order here: First, since $\bar{u}$ has to change its sign as the system crosses $\sigma(x)=0$, the sliding surface has to be a hyperplane (dimension of one less than the state space). Secondly, it is $\bar{u}$ which is mainly responsible for driving and keeping the system onto the sliding plane $\sigma(x)=0$ (where $\bar{u}=0$ as well). Provided that the gain $\eta^{2}$ has been chosen large enough, $\bar{u}$ can provide the required robustness due to momentary disturbances and unmodeled dynamics without any compromise in stability.

The linear feedback law (12) is designed such that the system has the desired dynamics on the sliding plane. Since $\sigma(\boldsymbol{x})=0$, in this case:

$$
u=\hat{u}=-\left(s^{T} b\right)^{-1} s^{T} A x
$$

and the closed-loop dynamics are given by

$$
\dot{x}=\left[A-b\left(s^{T} b\right)^{-1} s^{T} A\right] x
$$

or

$$
\dot{\boldsymbol{x}}=(\boldsymbol{A}-\boldsymbol{b} \boldsymbol{k}) \boldsymbol{x}
$$

where the gain vector $\boldsymbol{k}$ can be found from standard methods such as pole placement. The closed-loop dynamics matrix

$$
\boldsymbol{A}_{C}=\boldsymbol{A}-\boldsymbol{b} \boldsymbol{k}
$$

has eigenvalues specified for the desirable response. It should be mentioned that one of the eigenvalues of $A_{C}$ must be specified to be zero. This is consistent with our decomposition (11). The linear feedback $\hat{u}$ provides the desired dynamics on the sliding plane only. Therefore, $\hat{u}$ has no effect in a direction perpendicular to $\sigma(x)=0$. With $A_{C}$ specified and $k$ com- 
puted from pole placement, we can determine $s$ as follows: From (14) and (15):

$$
k=\left(\boldsymbol{s}^{T} b\right)^{-1} \boldsymbol{s}^{T} A
$$

and

$$
\boldsymbol{s}^{T}(\boldsymbol{A}-\boldsymbol{b k})=0 \quad \text { or } \quad \boldsymbol{s}^{T} A_{C}=0 .
$$

Therefore $s^{T}$ is found as a left annihilator of $A_{C}$, or $s$ is a right eigenvector of $\left(A_{C}\right)^{T}$, which corresponds to the zero eigenvalue. With this choice of $s$, the sliding plane $s^{T} x=0$ and the feedback control law (11) are completely determined. It should be pointed out that, in applications, the states $x_{1}$, $x_{2}$, and $x_{3}$ are to be interpreted as errors between the actual values of $q, \theta$, and $z$ and their set points.

The aforementioned procedure can be easily extended to the case of nonlinear systems, linear in the control effort of the form:

$$
\dot{\boldsymbol{x}}=f(x)+b(x) u .
$$

Design of the sliding-surface coefficients can be based on the linearized version of (18) as in (17). Then the nonlinear feedback control law,

$$
u=-\left(s^{T} b(x)\right)^{-1} s^{T} f(x)-\eta^{2}\left(s^{T} b(x)\right)^{-1} \operatorname{sign}(\sigma)
$$

should replace (11). In this work we designed and implemented the control law as in (11) based on the linear model, as if no information regarding nonlinear characteristics of the vehicle dynamics existed. This approach clearly constitutes a "worst case scenario" and enhances the demonstration of the robustness properties of the design.

\section{Sliding Mode with Adaptive Control}

In the previous section we addressed the problem of designing a robust controller for diving maneuvers based on dominant linear dynamics with known coefficients and knowledge of bounds on the uncertainties. However, the linearized behavior of the AUV (expressed by the $A, b$ matrices in the dynamics (2)) has characteristics changing with operating conditions such as speed and depth. This uncertain behavior can easily be included in the perturbation $f$ by increasing the magnitude of the coefficient $\eta^{2}$ of the nonlinear control term $\bar{u}$.

From a different perspective, we can make use of adaptive control techniques to compensate for the linear uncertainties while not affecting the nonlinear perturbation term $f$ and the switching effort $\eta^{2}$.

Let

$$
\boldsymbol{A}_{\boldsymbol{m}}=\left[\begin{array}{ccc}
-\alpha_{1} & -\alpha_{2} & -\alpha_{3} \\
1 & 0 & 0 \\
0 & -\bar{U}_{0} & 0
\end{array}\right], \quad \boldsymbol{b}=\left[\begin{array}{l}
b \\
0 \\
0
\end{array}\right]
$$

represent the desirable or nominal closed-loop dynamics of the system, with $v$ forward velocity, assumed to be known. Then the dynamics of the AUV in the vertical motion can be written as

$$
\dot{x}=A_{m} x+b\left(\delta+K^{T} x\right)+f
$$

with $K$ depending on the actual vehicle dynamics. On the basis of this model we can design an adaptive controller which yields a closed-loop stable behavior for any uncertainty on the vehicle dynamics. In particular, we can show the following:

a) Let $\left(\lambda, s^{T}\right)$ be a pair of real eigenvalue $(\lambda)$ and corresponding left eigenvector of the matrix $A_{m}$ in (20) such that $s^{T} b \neq 0$ (assume $s^{T} b>0$ ). The existence of $s$ is guaranteed by the pair $\left(A_{m}, b\right)$ being completely controllable [14].

b) Let a bound $\eta^{2}$ on the nonlinearity $f$ be defined by

$$
\eta^{2}(x)>\frac{\mid s^{T} f(x)}{\left|s^{T} b\right|} .
$$

c) Let bounds on the coefficients $K$ in (21) be defined by

$$
K_{i}^{m}<K_{i}<K_{i}^{M}
$$

with $K_{i}, i=1, \cdots, n$ the elements of $\boldsymbol{K}=$ $\left[K_{1}, \cdots, K_{n}\right]^{T}$.

Then the control input:

$$
\delta(t)=-\hat{\boldsymbol{K}}^{T}(t) \boldsymbol{x}(t)-\eta^{2}(\boldsymbol{x}) \operatorname{sgn}(\sigma(t))
$$

with

$$
\sigma(t)=s^{T} \boldsymbol{x}(t)
$$

and the adaptive gains

$$
\dot{\hat{\boldsymbol{K}}}(t)=-\gamma(\hat{\boldsymbol{K}}(t))-\mu \boldsymbol{x}(t) \sigma(t)
$$

with [15]

$$
\gamma_{i}(\hat{K})= \begin{cases}0, & \text { if } K_{i}^{m}<K_{i}<K_{i}^{M} \\ \alpha\left(\hat{K}_{i}(t)-K_{i}^{m}\right), & \text { if } K_{i}(t)<K_{i}^{m} \\ \alpha\left(\hat{K}_{i}(t)-K_{i}^{M}\right), & \text { if } K_{i}(t)>K_{i}^{M}\end{cases}
$$

$\alpha$ being a positive constant, is such that the closed-loop system is exponentially stable and

$$
\lim _{t \rightarrow \infty} x(t)=0
$$

for any initial conditions.

Proof: From (24) and (25) we can write (21) as

$$
\begin{aligned}
\dot{\sigma}(t)+\lambda \sigma(t)=\boldsymbol{s}^{T} \boldsymbol{b} \tilde{\boldsymbol{K}}(t)^{T} \boldsymbol{x}(t)+ & \boldsymbol{x}^{T} \boldsymbol{f}(\boldsymbol{x}) \\
& -\boldsymbol{s}^{T} \boldsymbol{b} \eta^{2}(\boldsymbol{x}) \operatorname{sgn}(\sigma(t))
\end{aligned}
$$

with $\tilde{\boldsymbol{K}}(t)=\boldsymbol{K}-\hat{\boldsymbol{K}}(t)$ the parameter error. Define the Lyapunov function:

$$
V(\sigma, \tilde{K})=\frac{1}{2}\left(\sigma^{2}+\frac{s^{T} b}{\mu} \tilde{K}^{T} \tilde{K}\right)
$$

and its time derivative along the trajectories of (29) can be computed as

$$
\begin{array}{r}
\dot{V}(\sigma, \tilde{\boldsymbol{K}})=-\lambda \sigma(t)^{2}-\left(\boldsymbol{s}^{T} \boldsymbol{b} \eta^{2}(\boldsymbol{x})-\boldsymbol{s}^{T} \boldsymbol{f}(\boldsymbol{x}) \operatorname{sgn}(\sigma(t))\right)|\sigma(t)| \\
-\frac{\boldsymbol{s}^{T} \boldsymbol{b}}{\mu} \tilde{\boldsymbol{K}}(t)^{T} \gamma(\hat{\boldsymbol{K}}(t))
\end{array}
$$


It is easy to see from the definition of $\gamma$ in (29) that the right-most term in (31) is such that:

$$
\tilde{K}(t)^{T} \gamma(\hat{K}(t)) \geq 0
$$

which yields $\dot{V} \leq 0$. Therefore $\tilde{\boldsymbol{K}}(t), \sigma(t)$ are uniformly bounded, and furthermore, $\sigma(t) \in L_{2}$, the set of signals square integrable. This fact and the boundedness of the derivative $\dot{\sigma}(t)$ implies that $\sigma(t) \rightarrow 0$ as $t \rightarrow \infty$. Finally, $\boldsymbol{x}(t) \rightarrow 0$ is guaranteed by the choice of a stable sliding surface.

The control law (24) is basically analogous to (11) with the addition of the adaptive gains $\hat{\boldsymbol{K}}(t)$ adjusted on-line on the basis of (26). They guarantee the convergence of the state vector to the sliding surface $s^{T} x=0$ in spite of uncertainties of the linear part of the vehicle dynamics. Notice that the nonlinear control part $\bar{u}$ is not affected by this uncertainty and is identical to (13).

\section{Sliding Mode Control With Estimated States}

The control structures addressed thus far require the signals in the state vector to be available for measurements. In terms of the dive maneuver of the AUV, this implies that pitch rate and pitch angle signals are measured by the respective gyros, while depth is measured by a pressure cell.

In some cases it might be desirable to design a control system based on the measurements of a restricted set of signals. This is typically the case in two situations: (a) When we want to reduce the number of sensors (gyros in this case), or (b) to improve the reliability of the system by the design of a controller which is robust in the presence of failures of some of the sensors.

Most approaches to the sliding mode control available in the literature are based on the assumption of full state feedback. In this section we address the problem of designing a controller based on the observed state, and we show that global stability can still be guaranteed for a class of nonlinearities of interest.

Consider still the system in (2) and assume we measure the signal $y$ (say, the depth error $\left(z-z_{d}\right)$ ) in the form:

$$
\left\{\begin{array}{l}
\dot{x}=A x+b u+f \\
y=c x
\end{array}\right.
$$

with $(A, b)$ a controllable pair, and $(A, c)$ an observable pair, and the perturbation $f(x)$ having a finite $L_{\infty}$ norm [16] as

$$
\|f(x(t))\| \leq\left|H_{1}\right|\left|x_{t}\right|
$$

where we define the $L_{\infty}$ norm:

$$
\left|\boldsymbol{x}_{t}\right|=\sup _{\tau \leq t}\|x(\tau)\|
$$

In this section we show that given an observer for (33):

$$
\dot{\hat{x}}=A \hat{x}+b u+K(y-c \hat{x})
$$

with $(\boldsymbol{A}-\boldsymbol{K} \boldsymbol{c})$ having eigenvalues in the stable region, we can design a sliding mode controller which guarantees global stability and $x(t) \rightarrow 0$, provided that the perturbation $f$ satisfies
(34) with $\left|H_{1}\right|$ small enough. In particular: (a) Let $\hat{x}$ be the estimated state defined by (36); (b) let:

$$
u(t)=-\boldsymbol{L} \hat{\boldsymbol{x}}(t)-\eta^{2} \operatorname{sgn}\left(s^{T} \hat{x}(t)\right)
$$

be the sliding mode control designed on the basis of the system,

$$
\dot{\hat{\boldsymbol{x}}}=\boldsymbol{A} \hat{\boldsymbol{x}}+\boldsymbol{b} u+\tilde{\boldsymbol{f}}
$$

with $\tilde{f}$ such that:

$$
\left\{\begin{array}{l}
\dot{\tilde{x}}=(\boldsymbol{A}-\boldsymbol{K} \boldsymbol{c}) \tilde{\boldsymbol{x}}+\boldsymbol{f} \\
\tilde{\boldsymbol{f}}=\boldsymbol{K} \boldsymbol{c} \tilde{\boldsymbol{x}} .
\end{array}\right.
$$

Note that since the linear mapping (39) is known and exponentially stable, if we know a bound on $f$ we can easily determine a bound on $\tilde{f}$ in (38) and design a sliding mode controller for (38); (c) let $f$ satisfy (34); Then there exists an $H^{*}$ such that:

$$
\lim _{t \rightarrow \infty} x(t)=0
$$

provided that $f$ is such that $\left|H_{1}\right|<H^{*}$.

Proof [10]: In order to show the results, define the state estimate error, $\tilde{\boldsymbol{x}}=\boldsymbol{x}-\hat{\boldsymbol{x}}$. Then it is easy to see that (38) and (39) are an equivalent state-space representation of the system (33) and its observer (36). Also, by the arguments in Section II applied to the system (39), the control input $u$ in (37) ensures that the estimated state $\hat{x}$ tends to the sliding surface $s^{T} \hat{x}=0$ and then:

$$
\lim _{t \rightarrow \infty} \hat{x}(t)=0 .
$$

Therefore we can look at the mapping (39) as a feedback connection as in Fig. 2, with $H_{0}$ representing the linear mapping $H_{0}: f \rightarrow \tilde{f}$ in (39), and $H_{1}: x \rightarrow f$ the nonlinear perturbation. By the small gain theorem [16] we can see that the system $\hat{x} \rightarrow \boldsymbol{x}$ is BIBO stable, provided that $\left|H_{0}\right|\left|H_{1}\right|<1$, which proves the results.

This result shows that a control system designed on the sliding mode approach using an estimated state is still stable provided that the perturbations in its dynamics are "small enough" in their $L_{\infty}$ norm. Greater degrees of robustness can be achieved by the use of robust observers such as the class introduced in [17], provided that the entry point of the perturbations is known. This is the case, for example, of a system with dynamical equations:

$$
\left\{\begin{array}{l}
\dot{x}=A x+b(u+f) \\
y=c x .
\end{array}\right.
$$

In this case it will be shown in a forthcoming paper [10] that a class of observers can be constructed with an arbitrary degree of robustness.

\section{Simulation Results}

The sliding mode compensator was designed along the lines of Section II based on the nominal linear model (33) and for the nominal speed of $6 \mathrm{ft} / \mathrm{s}$. Closed-loop poles -0.25 and -0.27 were selected for the system on the sliding plane with 


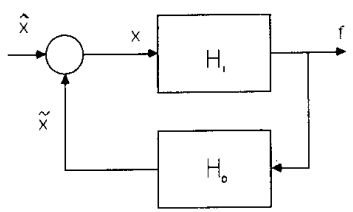

Fig. 2. Error model for the vehicle with observer.

the third pole at zero. The poles chosen for the observer were $-4.5,-4.75$, and -4.95 . Equation (17) then determined the sliding plane, and (11) the control law:

$$
u=-5.1429 \hat{q}+1.0714 \hat{\theta}+k_{n} \operatorname{satsgn}(\sigma)
$$

where

$$
k_{n}=\eta^{2}\left(s^{T} b\right)^{-1}
$$

is the nonlinear gain, and we use

$$
\operatorname{satsgn}(\sigma)= \begin{cases}+1, & \text { if } \sigma \geq \phi \\ -1, & \text { if } \sigma \leq \phi \\ \sigma / \phi, & \text { if }-\phi<\sigma<\phi\end{cases}
$$

instead of the pure switch sign $(\sigma)$ in order to avoid numerical chattering. Another saturation function was introduced to limit the total dive plane angle between -0.4 and $+0.4 \mathrm{rad}$. Simulated responses for both the linearized and nonlinear model under sliding mode control using $\left(k_{n}, \phi\right)=(4,0.4)$ are shown in Fig. 3 for a depth command of $100 \mathrm{ft}$. Further details can be found in [18].

Comparison of the two responses demonstrates the expected ability of the sliding mode compensator to handle unmodeled nonlinearities. It is interesting to note that since the nonlinear vehicle experiences speed loss while diving, it switched its dive planes from +0.4 to -0.4 earlier than the linear model.

The next test for the compensator was its robustness to parameter variations and unmodeled dynamics. Sliding mode controllers are expected to outperform more conventional linear state feedback schemes with respect to their robustness. This is true for the following two reasons: First, choosing $\eta^{2}$ sufficiently large will guarantee that the system approaches the sliding surface even for the off-design case. Secondly, since at the final stage the system evolves in a lower dimension state space (the sliding surface), it is naturally more robust than the original higher dimensionality system. Fig. 4 shows a comparison of actual depth responses for the following sets of pitch dynamics:

$$
\begin{aligned}
& \text { Case (a) }: \dot{q}=-0.7 q-0.03 \theta-1.00 \times 0.035 \delta \\
& \text { Case (b) }: \dot{q}=-.07 q-0.03 \theta-0.50 \times 0.035 \delta \\
& \text { Case (c) }: \dot{q}=-0.7 q-0.03 \theta-0.25 \times 0.035 \delta \\
& \text { Case (d) }: \dot{q}=-0.7 q-0.03 \theta-6.00 \times 0.035 \delta
\end{aligned}
$$

Control design was based on the nominal case (a) with $\left(k_{n}, \phi\right)=(5,0.2)$, closed-loop poles at $(0,-0.65,-0.69)$, and observer poles at $(-6.57,-6.85,-7.13)$. As can be seen from Fig. 4, even under a $24: 1$ change in the coefficient of
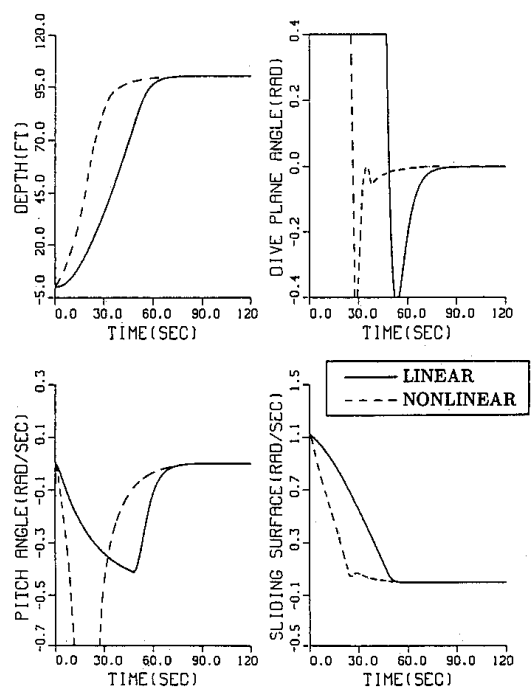

Fig. 3. Dynamic responses of the linear and nonlinear models.

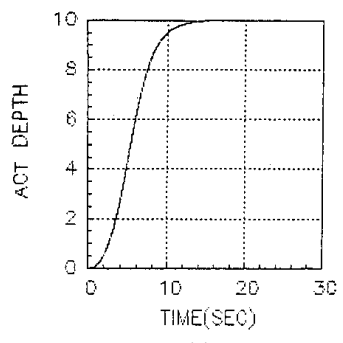

(a)

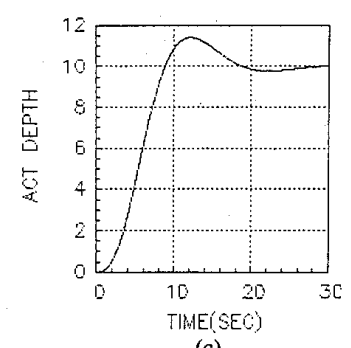

(c)

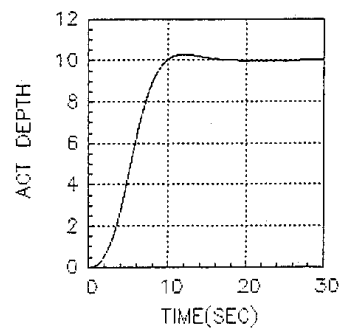

(b)

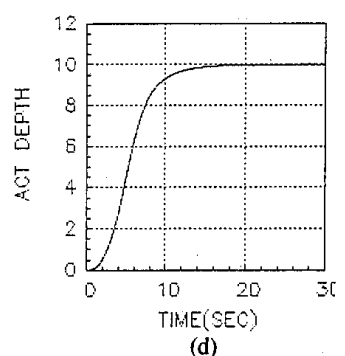

(d)
Fig. 4. Robustness test for the linear system.

the $b$ matrix the vehicle response remains stable, faster, or slower as expected.

Analogous robustness tests were performed by applying the nominal control law (40) to the nonlinear vehicle model under a wide variation in certain essential hydrodynamic and hydrostatic parameters. Simulation results are presented in Figs. 5-7 for the following system/model mismatch cases:

Fig. 5: $M_{\delta} \times 2.0$

Fig. 6: $M_{q} \times 0.5, \quad M_{\delta} \times 0.5, \quad\left(z_{G}-z_{B}\right) \times 0.5$

Fig. 7: $\left(z_{G}-z_{B}\right) \times 0.25$.

These coefficients were selected in view of their significance in the vehicle dynamic response. The rotary damping coefficient $M_{q}$ affects the hydrodynamic moment of the vehicle in the 

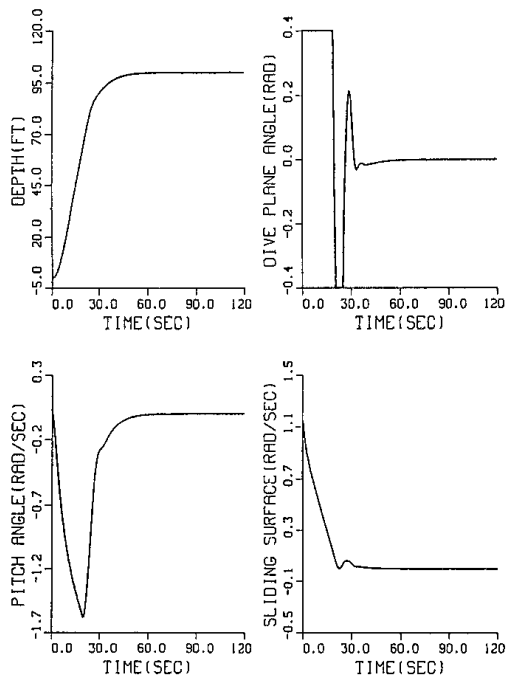

Fig. 5. First robustness test for the nonlinear system.
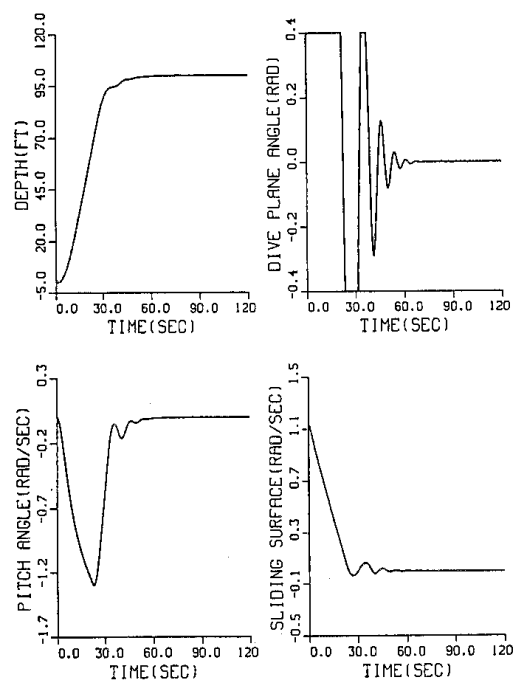

Fig. 6. Second robustness test for the nonlinear system.

vertical plane. The center of gravity/center of buoyancy separation $z_{G}-z_{B}$ represents the hydrostatic restoring moment. The hydrodynamic coefficient $\boldsymbol{M}_{\delta}$ is proportional to the pitch moment generated by the dive planes and is directly related to the gain margin of the system. As can be seen from the simulation results, the compensator provided stable response and performed consistently at different parameter values and coefficient mismatch.

The adaptive algorithm presented in the previous sections has been simulated in several diving maneuvers, using the model developed in [2], [3], [12]. Figs. 8 and 9 compare actual and desired depth at 500 and $300 \mathrm{rpm}$, together with the adaptive gains and the signal $\sigma=s^{T} x$ in (25) driving the controller. From these and various simulations it can be seen that the tracking of the depth signal is obtained for different operating conditions. The adaptive gains provide the necessary stabilizing action, together with the variable structure input.
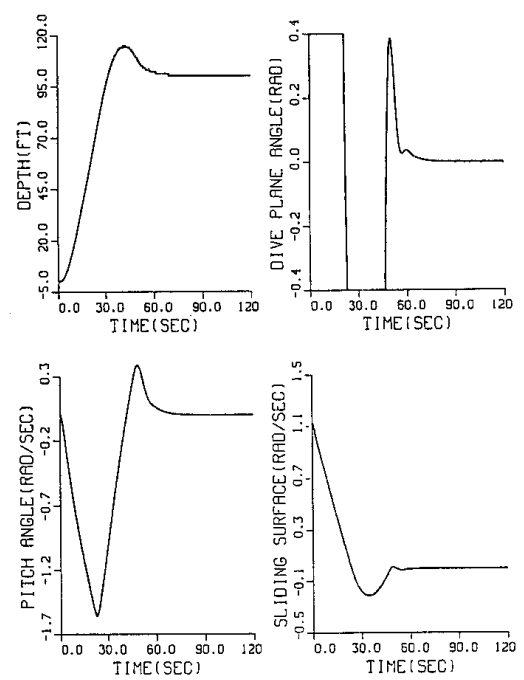

Fig. 7. Third robustness test for the nonlinear system
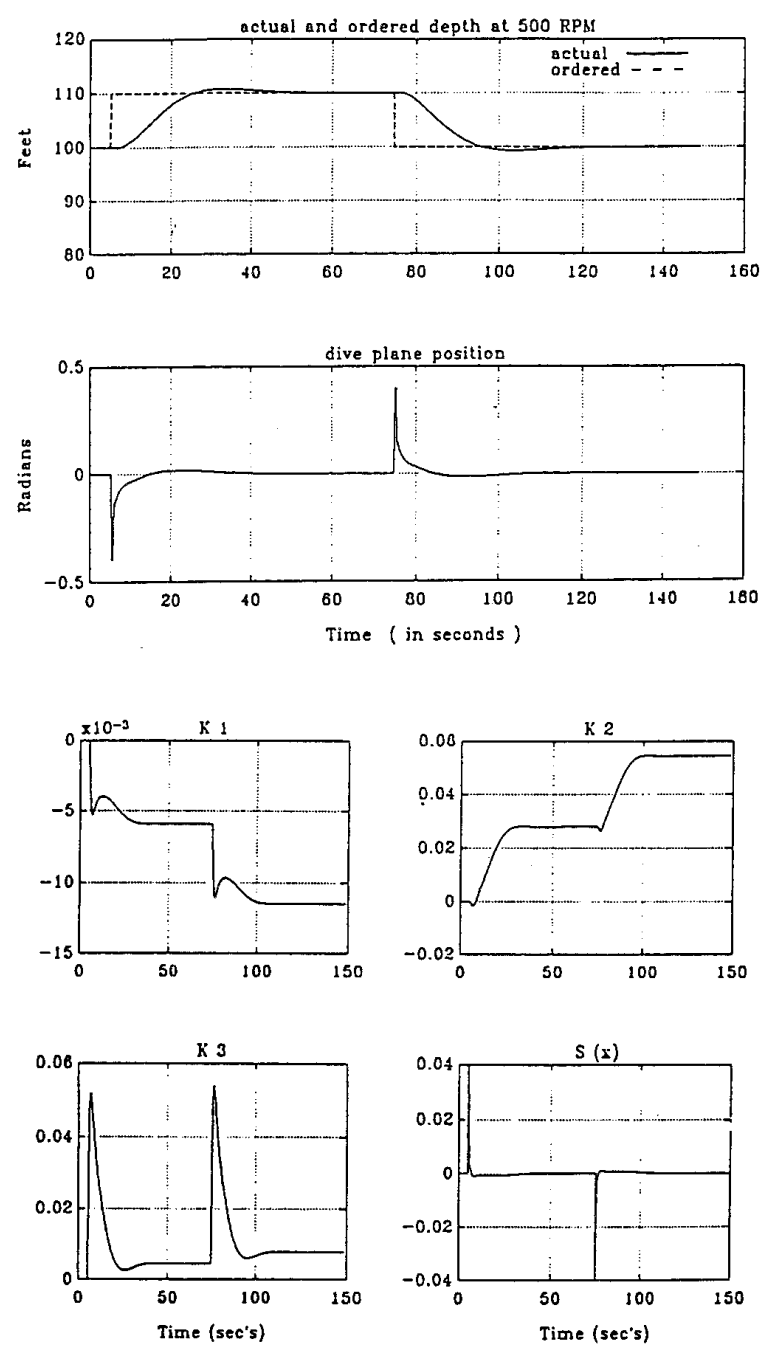

Fig. 8. Vehicle depth and adaptive gains at $500 \mathrm{rpm}$. 

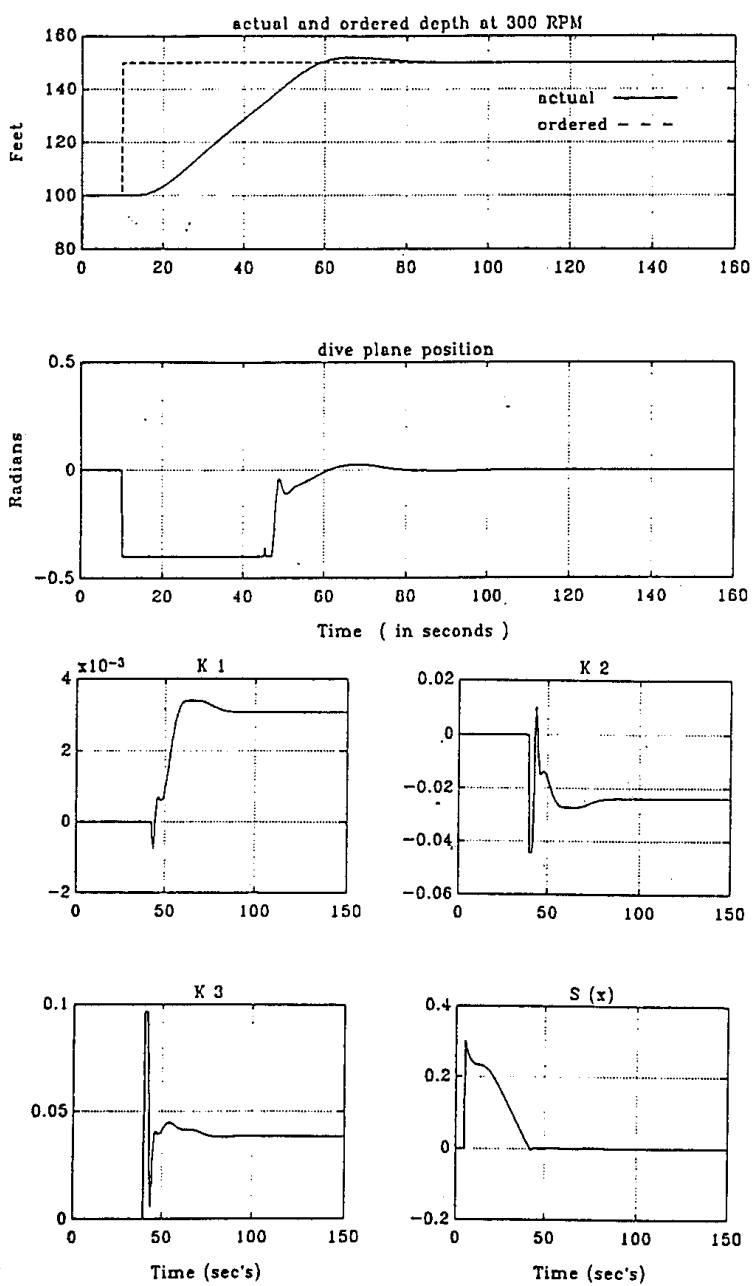

Fig. 9. Vehicle depth and adaptive gains at $300 \mathrm{rpm}$.

\section{Conclusions}

A control algorithm for the diving maneuver of a submersible vehicle has been presented. Its main feature is that it combines the adaptivity of a direct adaptive control algorithm with the robustness of a sliding mode controller. At the same time, its implementation is simple enough to be attractive for on-line realization using commercial microprocessors. Also, it is shown that the controller presented in its nonadaptive form can be combined with a standard Luenberger observer designed on the basis of a nominal dynamic model.

\section{ACKNOWLEDGMENT}

The authors would like to recognize the technical support of H. Cook at the Naval Surface Warfare Center, White Oak Detachment.

\section{REFERENCES}

[1] M. Gertler and G. R. Hagen, "Standard equations of motion for submarine simulations," Naval Ship Research and Development, NSRD Rep. 2510, 1967.

[2] R. J. Boncal, "A study of model based maneuvering controls for autonomous underwater vehicles," Mechanical engineer's thesis, Naval Postgraduate School, Monterey, CA, 1987.
[3] N. S. Smith, J. W. Crane, and D. C. Summey, "SDV simulator hydrodynamic coefficients," NCSC Tech. Memo. 231-78, 1984.

[4] K. Larsen, "Reduced hydrodynamic modeling for a submersible vehicle," Master's thesis, Naval Postgraduate School, Monterey, CA, 1988 .

[5] J. J. E. Slotine and S. S. Sastry, "Tracking control of nonlinear systems using sliding surfaces, with application to robot manipulators," Int. $J$. Contr., vol. 38, no. 2, pp. 465-492, 1983.

[6] D. Yoerger and J. J. E. Slotine, "Robust tracking control of underwater vehicles," IEEE J. Oceanic Eng., vol. OE-10, pp. 462-470, Oct 1985.

[7] J. Y. S. Luh, M. W. Walker, and R. P. Paul, "Resolved acceleration control of mechanical manipulators," IEEE Trans. Automat. Contr. vol. AC-25, pp. 468-474, 1980.

[8] M. A. Schwartz, "Kalman filtering for adaptive depth, steering and roll control of an autonomous underwater vehicle," Master's thesis, Naval Postgraduate School, Monterey, CA, 1989.

[9] A. J. Healey, F. Papoulias, and R. Cristi, "Design and experimental verification of a model based compensator for AUV depth control," in Proc. 6th Int. Unmanned, Untethered, Submersible Techn. (Ellicott City, MD), June 1989, pp. 458, 474.

[10] R. Cristi, A. J. Healey, and F. Papoulias, "Dynamic output feedback by robust observer and variable structure control," in Proc. 1990 Amer. Contr. Conf. (San Diego, CA), May 1990, to be published.

[11] R. A. DeCarlo, S. H. Zak, and G. P. Matthews, "Variable structure control of nonlinear multivariable systems: A tutorial," IEEE Proc., vol. 76, Mar. 1988, pp. 212-232.

[12] M. Schwartz and R. Cristi, "Indirect adaptive control of an AUV," 
Elec. and Comput. Eng. Dept., Naval Postgraduate School, Monterey, CA, NPS Tech. Rep., Mar. 1989.

[13] E. A. Barbashin, Introduction to the Theory of Stability. Groningen, The Netherlands: Wolters-Noordhoff, 1970.

[14] T. Kailath, Linear Systems. Englewood Cliffs, NJ: Prentice-Hall, 1980.

[15] P. A. Ioannou and K. S. Tsakalis, "A robust direct adaptive controller," IEEE Trans. Automat. Contr., vol. AC-31, pp. 1033-1043, Nov. 1986.

[16] C. A. Desoer and M. Vidyasagar, Feedback Systems: Input-Output Properties. New York: Academic, 1975.

[17] J. C. Doyle and G. Stein, "Robustness with observers," IEEE Trans. Automat. Contr., vol. AC-24, pp. 607-611, Aug. 1979.

[18] J.-N. Sur, "Design and investigation of a dive plane sliding mode compensator for an autonomous underwater vehicle," Master's thesis, Naval Postgraduate School, Monterey, CA, 1989.

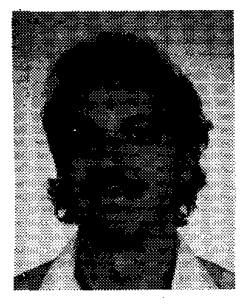

Roberto Cristi (S'82-M'83) was born in Ferrara, Italy, on September 3, 1952. He graduated (maxima cum laude) in electrical engineering from the University of Padua (Italy) in 1977, received the M.Sc degree from UMIST, Manchester, and Brunel University, Uxbridge (UK), and the Ph.D. degree from the University of Massachusetts at Amherst in 1983, all in electrical engineering.

He has been with the University of Padua, Italy, the General Electric Company, Leicester (UK), and the University of Michigan, Dearborn. Also, he has been a Consultant for the Ford Motor Company. At the present time he is an Associate Professor in the Electrical and Computer Engineering Department of the Naval Postgraduate School in Monterey, CA. His professional interests are in adaptive control, image processing, and signal processing.

Dr. Cristi is a member of Tau Beta Pi and Sigma Chi.

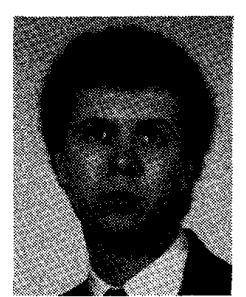

Fotis A. Papoulias received the Diploma from the Technical University of Athens, Greece, in 1983 and the M.S.E. and Ph.D. degrees from the University of Michigan, Ann Arbor, in 1986 and 1987, respectively.

He is currently with the Mechanical Engineering Department at the Naval Postgraduate School, Monterey, CA. His primary research interests are in the areas of nonlinear dynamics and control.

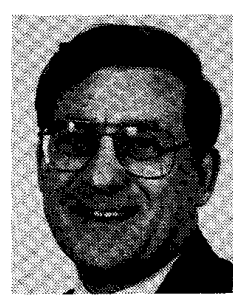

Anthony J. Healey was graduated from London and Sheffield Universities with the B.Sc.(eng.) and Ph.D. degrees in mechanical engineering in 1961 and 1966 , respectively.

He emigrated to the United States in 1966 and has taught at the Pennsylvania State University, the Massachusetts Institute of Technology, becoming Full Professor of Mechanical Engineering in 1974 at the University of Texas at Austin. In 1981 he joined Brown and Root Inc. as Manager of the Pipeline and SubSea Technology Research Group, leaving in 1986 to take up his present position as Professor and Chairman of Mechanical Engineering at the U.S. Naval Postgraduate School (NPS). His areas of specialty include mechanical system dynamics, vibration, and control systems and he is presently the Leader of an interdisciplinary project in mission planning, naviagation, and control for Autonomous Underwater Vehicles at NPS. 